\title{
New insight into shocks and star formation in Orion: First light with GriF`
}

\author{
M. Gustafsson ${ }^{1}$, L. E. Kristensen ${ }^{1}$, Y. Clénet ${ }^{2}$, D. Field ${ }^{\star \star, 1}$, J. L. Lemaire ${ }^{\star \star, 3,4}$, G. Pineau des Forêts ${ }^{5}$, \\ D. Rouan ${ }^{2}$, and E. Le Coarer ${ }^{6}$
}

${ }^{1}$ Department of Physics and Astronomy, University of Aarhus, 8000 Aarhus C, Denmark

2 Observatoire de Paris-Meudon, LESIA, 92195 Meudon Principal Cedex, France

3 Observatoire de Paris-Meudon, LERMA and UMR8112 of the CNRS, 92195 Meudon Principal Cedex, France

${ }^{4}$ Université de Cergy-Pontoise, LERMA and UMR8112 of the CNRS, 95806 Cergy Cedex, France

${ }^{5}$ Institut d'Astrophysique Spatiale, Université Paris XI, 91405 Orsay Cedex, France

${ }^{6}$ Observatoire de Grenoble, Laboratoire d'Astrophysique de l'Observatoire de Grenoble, France

Received 20 January 2003 / Accepted 30 June 2003

\begin{abstract}
A new instrument, "GriF", has been used on the Canada-France-Hawaii Telescope to record spatially and velocity resolved images, in the $\mathrm{H}_{2} v=1-0 \mathrm{~S}(1)$ line in the $K$-band, of a $1^{\prime} \times 1^{\prime}$ field in the core of the Orion Molecular Cloud, OMC1. Using the PUEO adaptive optics system combined with Fabry-Perot interferometry, diffraction limited spatial resolution of 0. ' 15 and velocity discrimination of $\sim 1 \mathrm{kms}^{-1}$ has been achieved. These data yield graphic images of shocks in OMC1, presenting a level of detail which has not previously been attained. Observed structures suggest that a significant fraction of the regions strongly emitting in $\mathrm{H}_{2}$ do so through local, internal shocks, associated with star formation within the clumps of gas involved. Thus the core of $\mathrm{OMC} 1$ is revealed for the first time as a region which contains a number of developed protostars.
\end{abstract}

Key words. ISM: individual objects: OMC1 - ISM: kinematics and dynamics - ISM: molecules - shock waves - ISM: lines and bands - stars: formation

\section{Introduction}

The Orion Nebula Cluster (ONC), which contains the Trapezium stars, and the Orion molecular cloud (OMC1) associated with $\mathrm{ONC}$, provide a rich source of information on young stellar objects, through a plethora of studies mostly in the visible but ranging from X-ray to radio wavelengths (Ferland 2001; O’Dell 2001; Luhman et al. 2000). Data record the presence of many protostars, outflows and larger scale flows, showing for example the explosive character of the Becklin-Neugebauer-IRc2 complex (BN-IRc2) embedded in OMC1 (Allen \& Burton 1993; Schild et al. 1997; Chen et al. 1998; Stolovy et al. 1998; Schultz et al. 1999).

An enhanced appreciation of the processes at work in Orion has been provided by images resolved in radial velocity, for example in the near-infrared (NIR) (Sugai et al. 1995, Chrysostomou et al. 1997; Salas et al. 1999), which, with

Send offprint requests to: D. Field,

e-mail: dfield@phys.au.dk

* Based on observations performed at the Canada-France-Hawaii Telescope, Mauna Kea, Hawaii. CFHT Corporation.

$\star \star$ Visiting astronomer, Canada-France-Hawaii Telescope Corporation, operated by the National Research Council of Canada, the Centre National de la Recherche de France and the University of Hawaii. proper motion studies (e.g. Doi et al. 2002), reveal gas with relative velocities ranging from $10 \mathrm{kms}^{-1}$ to several hundred $\mathrm{kms}^{-1}$ within ONC and in the outer reaches of OMC1. Here we report the highest spatial resolution infrared images yet acquired, which also include velocity resolution. Combining Fabry-Perot interferometry with adaptive optics on the CanadaFrance-Hawaii Telescope (CFHT), in first light on a new instrument ("GriF"), we have obtained spatially and velocity resolved images of the inner $1^{\prime} \times 1^{\prime}$ region of OMC1. These data show the gas kinematics and morphology of numerous energetic zones, recorded using the $\mathrm{H}_{2} v=1-0 \mathrm{~S}(1)$ line at $2.121 \mu \mathrm{m}$. Emission in this line traces supersonic motions which generate shocks and vibrationally excited $\mathrm{H}_{2}$. The high spatial resolution of the present observations allows visualization of shocks in a manner which has not previously been possible. Detailed association can be made between gas velocity and $\mathrm{H}_{2}$ emission brightness, providing key data for theoretical models of shocks and star formation. Consideration of the morphology of 19 shocked zones suggests that the emission observed arises in part from outflows from protostars already present within dense clumps of gas, rather than purely from external impact by fast outflows from BN-IRc2, which is the current model (Vannier et al. 2001). The present observations yield a new view of early star formation in OMC1 and may 

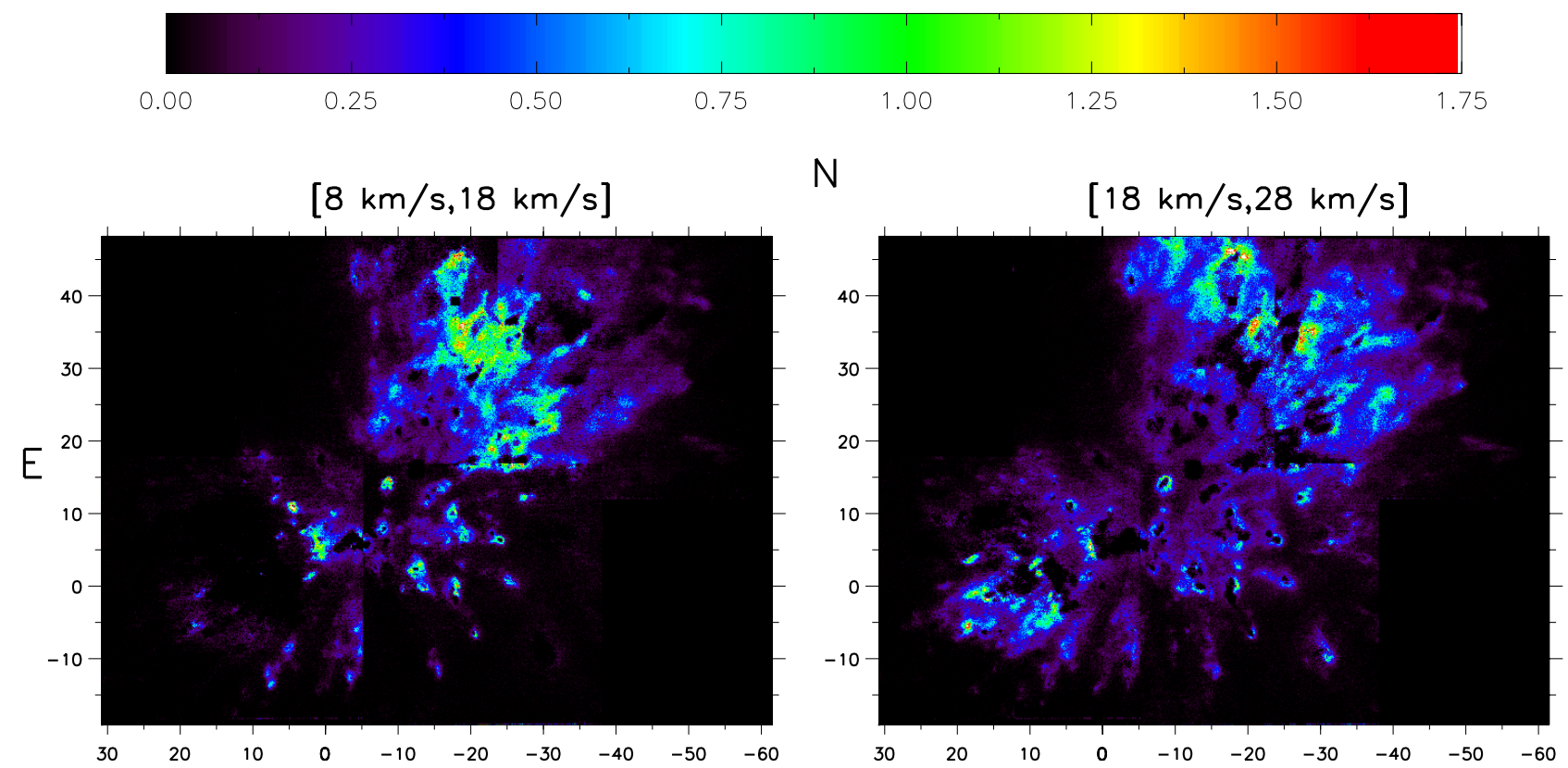

Fig. 1. The field in OMC1 covered in the present observations. The position $(0,0)$ is that of TCC0016 $05^{\mathrm{h}} 35^{\mathrm{m}} 14.91,-05^{\circ} 22^{\prime} 399^{\prime} 31(\mathrm{~J} 2000)$. Data show emission in the $\mathrm{H}_{2} \mathrm{~S}(1) v=1-0$ line for the velocity channels integrated over velocities between $v_{\mathrm{lsr}}=18$ to $28 \mathrm{~km} \mathrm{~s}$ s $^{-1}$ (right-hand panel) and 8 to $18 \mathrm{~km} \mathrm{~s}^{-1}$ (left-hand panel). The values on the colour bar in this and all subsequent figures represent counts per pixel per second. See text for calibration to absolute brightness. Axes are labelled in arcseconds.

create a new perspective on star formation in the vicinity of massive stars.

\section{Observations and data reduction}

OMC1 was observed using the CFHT on the night of December 5th 2000. The GriF instrument, described in detail elsewhere (Clénet et al. 2002), is a combination of the PUEO adaptive optics (AO) system on the CFHT with interferometric spectral scanning, with the option (not implemented here) of inclusion of a grism. The interferometer is a Queensgate ET50WF Fabry-Perot, affording a measured spectral resolution $\lambda / \Delta \lambda \sim 2000$, that is, $150 \mathrm{kms}^{-1}$. Observations were performed in the NIR in the $K$-band of the strongest $\mathrm{H}_{2}$ emission line in Orion, $v=1-0 \mathrm{~S}(1)$, at a rest wavelength of $2.1212544 \mu \mathrm{m}$ (Bragg et al. 1982). Recent NIR studies of Orion using spectroimaging have involved higher spectral resolution, of respectively $14 \mathrm{~km} \mathrm{~s}^{-1}$ (Chrysostomou et al. 1997) and $24 \mathrm{~km} \mathrm{~s}^{-1}$ (Salas et al. 1999), but both studies suffer from a lack of spatial resolution, which lay between 1'.65 and 2'. 0 . The PUEO adaptive optics system in the present work yielded spatial resolution using GriF of $\sim 0$. $^{\prime} 15$, with a pixel scale of $0{ }^{\prime} 035$.

The region of OMC1 observed consists of four overlapping fields each $36^{\prime \prime} \times 36^{\prime \prime}$, with the entire region centered approximately $15^{\prime \prime} \mathrm{N}$ and $15^{\prime \prime} \mathrm{W}$ of TCC0016 $\left(05^{\mathrm{h}} 35^{\mathrm{m}} 14^{\mathrm{s}} .91\right.$, $-05^{\circ} 22^{\prime} 39^{\prime} 31$ (J2000)), which we use as a positional reference throughout, lying itself about $40^{\prime \prime} \mathrm{N}$ and $15^{\prime \prime} \mathrm{W}$ of the Trapezium cluster. We designate these four fields as SE, NE, SW, NW. The extent of the field covered may be seen in Fig. 1. Observations proceeded by setting the Fabry-Perot to a wavelength far in the wings of any $\mathrm{H}_{2}$ emission and for each region progressively scanning through the $\mathrm{H}_{2}$ line, using step sizes varying between $4.4 \times 10^{-4} \mu \mathrm{m}$ and $4.6 \times 10^{-4} \mu \mathrm{m}$, that is, $\sim 65 \mathrm{kms}^{-1}$. To prevent the superposition of different FP orders during scanning, a $\mathrm{H}_{2} v=1-0 \mathrm{~S}(1)$ interference filter with a central wavelength of $2.1222 \mu \mathrm{m}$, bandwidth $0.02 \mu \mathrm{m}$, was inserted between the FP and the detector. For each wavelength of interest and each region, a single exposure of $400 \mathrm{~s}$ was performed, forming in each case a "channel map". The 4 fields were scanned over different ranges about the rest wavelength, with between 8 and 12 channel maps obtained for each field. The minimum velocity range scanned was $433 \mathrm{~km} \mathrm{~s}^{-1}$ (nominally $2.1186 \mu \mathrm{m}$ to $2.12166 \mu \mathrm{m}$ ), and the maximum $678 \mathrm{kms}^{-1}$ (nominally $2.1194 \mu \mathrm{m}$ to $2.1242 \mu \mathrm{m}$ ), where all velocities refer to the radial component of motion. The choice of wavelength range was made through real-time observation of channel maps and through the time constraint that we sought to obtain the full set of data in a single night under the same stable prevailing weather conditions.

Various wavefront reference stars, necessary for functioning of the PUEO-AO system, were used: TCC0016 (12th magnitude), Paranego 1838 (8th magnitude) and Paranego 1819 (12th magnitude), appropriate to the fields involved. The estimate of spatial resolution of $0 . ' 15$ was obtained from the point spread function of stars within the fields observed. This spatial resolution was maintained throughout all observations. No deconvolution procedures were carried out on the data.

Calibration of GriF (Clénet et al. 2002) was performed before and after each set of channel maps for each of the four regions. Calibration covers (i) wavelength calibration by scanning through the $2.06163 \mu \mathrm{m}$ Ar line using an Ar lamp, with a step-size of $4.21 \times 10^{-4} \mu \mathrm{m}$ and (ii) flat-fielding to account for different sensitivities of each pixel of the detector, using a halogen lamp white light source at the same set of wavelengths as 
used in the observations. The scan over the Ar line also yields a phase-map of the instrument which allows a $2 \mathrm{D}$ correction to be applied pixel by pixel to the observational data. Other corrections to the raw data involve dark and bias subtraction, including bad pixel rejection. The sky background is obtained from data in the far wings of the $\mathrm{H}_{2}$ line. In $\mathrm{OMC} 1$, this background is small compared with signals in the $\mathrm{H}_{2}$ emission line and problems such as temporal variability of background do not arise. Telluric lines which may vary with time, specifically $\mathrm{OH}$ Meinel band emission, are not troublesome in the wavelength region of interest here. Conversion of count rates to absolute values of surface brightness was obtained by comparison with calibrated data in Vannier et al. (2001). Thus the count rate of 3.43 counts per second per pixel, at the peak of emission to the SE of TCC0016, corresponds to a velocity integrated brightness of $3.0 \pm 0.15 \times 10^{-5} \mathrm{~W} \mathrm{~m}^{-2} \mathrm{sr}^{-1}$. This conversion may be applied to all regions with the caution that systematic errors may arise through the differing conditions of airmass and of the atmosphere prevailing at the times of observation. For this reason, brightness is recorded in terms of count rate throughout.

If we choose a specific position on the sky and take a cut through the channels maps, integrating over $5 \times 5$ pixels (resulting in little loss of spatial resolution), we obtain a set of values of count rate to which may be fitted a lorentzian (Clénet et al. 2002; Chrysostomou et al. 1997; Salas et al. 1999). Taking a moving average of $5 \times 5$ pixels and obtaining fits moving from pixel to pixel, very precise fits may be found for the brighter objects. Including statistical error in the count rates, relative line centre wavelengths may be found in local zones, extending over at least a few arcseconds, to 2 to 3 parts per $10^{6}(3 \sigma)$, that is, to better than $\pm 1 \mathrm{~km} \mathrm{~s}^{-1}$. For weakly emitting regions, uncertainties of several $\mathrm{km} \mathrm{s}^{-1}$ are found.

Figure 2 shows the peaks of normalized Lorentzians fitted to channel map data, making a cut in the plane of the sky moving south to north through bright emission between two positions $15^{\prime \prime} 9 \mathrm{E}, 22^{\prime \prime} 0 \mathrm{~S}$ to $15^{\prime \prime} .9 \mathrm{E}, 1^{\prime \prime} .5 \mathrm{~S}$ relative to TCC0016 (Fig. 1). Peak positions march monotonically in velocity to progressively less blue-shifted values, ranging here over $\sim 10 \mathrm{~km} \mathrm{~s}^{-1}$. Progressing further north, we find that the velocity smoothly turns back upon itself from the maximum redshift shown in Fig. 2. This behaviour is omitted for clarity. The relative velocities of widely separated high brightness regions within any frame should in principle be as well-determined as local relative velocities, through application of the phase map, but we do not pursue proof of this further here.

In order to amalgamate the four fields into a single data cube, overlapping regions have been used to achieve consistency between wavelengths in the different fields. This involved some small wavelength shifts in the SW and NE regions to bring them to the same wavelength values. Absolute calibration is however not material to the present work and, for consistency with other data for Orion, we have chosen to assign to the mean velocity of all our observations a value of $12 \pm 6 \mathrm{~km} \mathrm{~s}^{-1}$, in the local standard of rest, $v_{\mathrm{lsr}}$, as provided through data in Chrysostomou et al. (1997), Salas et al. (1999) and O'Dell (2001). All values of velocity quoted or shown in

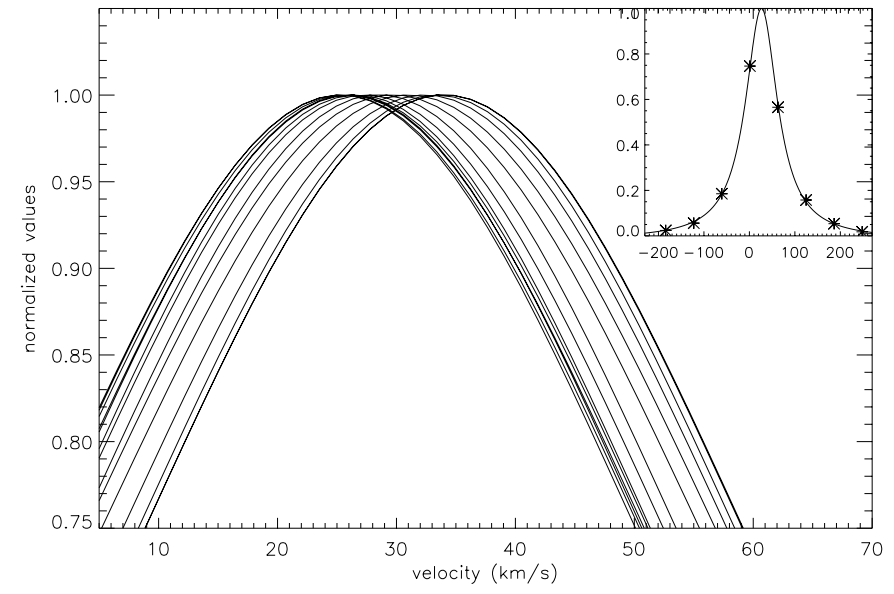

Fig. 2. A set of normalized lorentzian curves, zooming in on the peak, obtained by fitting to sets of count rates in the $\mathrm{S}(1) v=1-0 \mathrm{H}_{2}$ line, averaged over $5 \times 5$ pixels, for 9 velocity channels in the SE region. These results correspond to 14 steps, each of 1 pixel ( $\left.00^{\prime} 035\right)$, moving from south to north (left to right) over an angular range of 0.5 , in a brightly emitting region starting at position $15^{\prime \prime} 9 \mathrm{E}, 22^{\prime \prime} 0 \mathrm{~S}$ relative to TCC0016, the 0, 0 position in Fig. 1. The insert shows a typical fit obtained to a set of normalized count rates vs velocity in $\mathrm{km} \mathrm{s}^{-1}$.

figures are $v_{\text {lsr }}$ and all wavelengths are nominal wavelengths as measured.

In the treatment of the data described above, all regions are rejected for which no peak can be found in data for a cut through the velocity channels. If a double peak (say) is encountered, then we record a fit only to the stronger of the two peaks. This procedure rejects relatively weakly emitting fast moving objects superimposed on slower brighter objects. An example of such a region is the "bullet" 24 ". $2 \mathrm{~W} 6$ ". $5 \mathrm{~N}$ of TCC0016, whose velocity profile, taken from Chrysostomou et al. (1997) and shown in Stolovy et al. (1998), has a strong low velocity component and a weak high velocity component differing by $80 \mathrm{~km} \mathrm{~s}^{-1}$. Emission at this position is indeed observed in the high velocity channel map centered around a nominal wavelength of $2.119909 \mu \mathrm{m}$. However, the results presented below refer to low velocity objects only and exclude fast moving $\mathrm{H}_{2}$ emitting bullets, known to lie within our field (Chrysostomou et al. 1997; Stolovy et al. 1998; Salas et al. 1999; Lee \& Burton 2000).

\section{Results and discussion}

Figure 1 illustrates the nature of the GriF data, analyzed as in the preceding section, showing two adjacent velocity channels, each of $10 \mathrm{~km} \mathrm{~s}^{-1}$ extent. Markedly different structures are visible in these two channels. For example, strong emission in SE in channel $18-28 \mathrm{~km} \mathrm{~s}^{-1}$ is largely absent in channel 8$18 \mathrm{~km} \mathrm{~s}^{-1}$.

In order to convey the overall velocity structure in the region observed, we have prepared a velocity map of the entire zone, shown in Fig. 3. In preparing this map, all data involving pixels with velocity-integrated count rates below 0.3 counts per pixel per second, that is $\sim 10 \%$ of the maximum count rate, were rejected. 


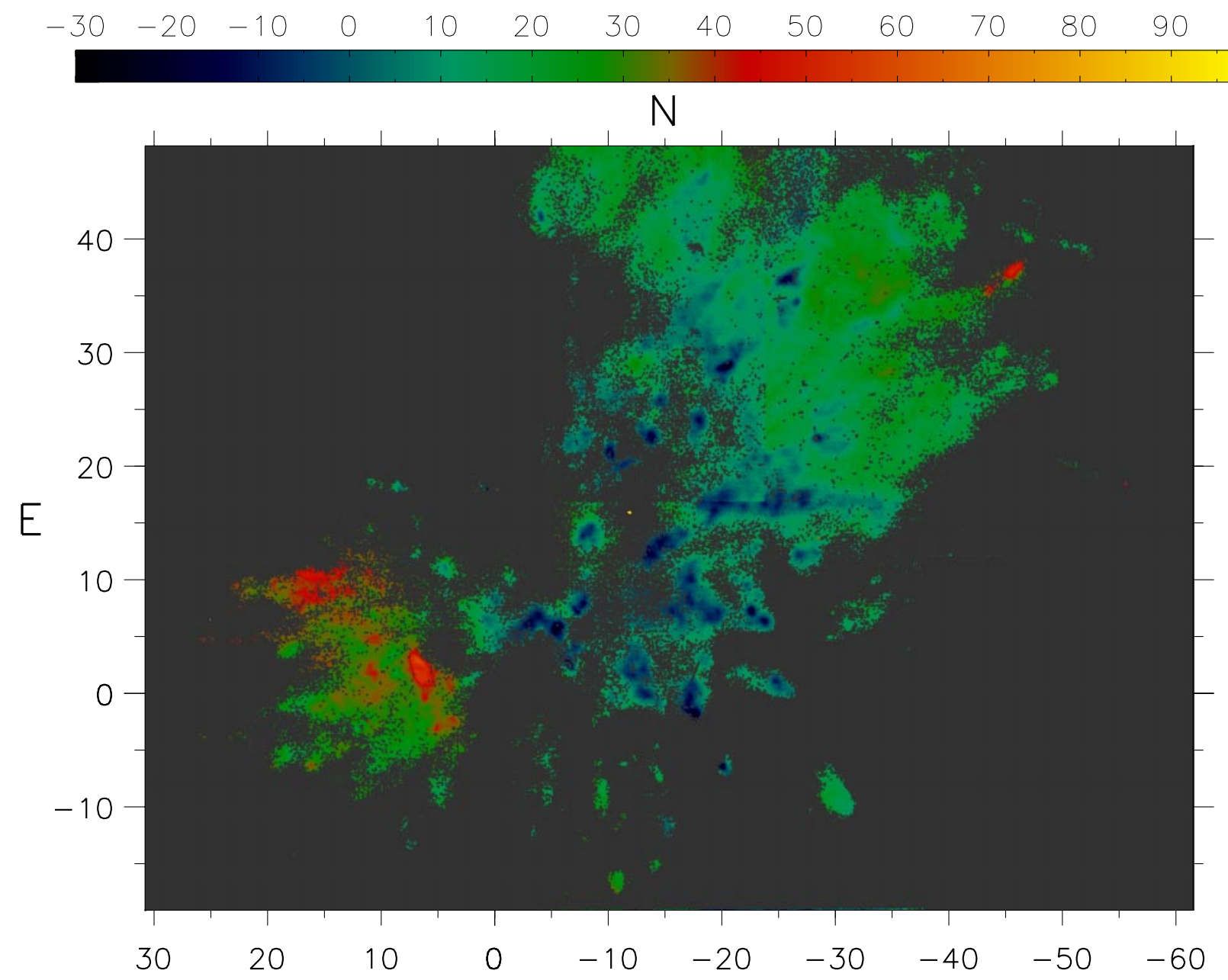

Fig. 3. The velocity field recorded for the full observed field in the direction of OMC1, using data reduction techniques described in Sect. 2. (0, 0 ) is the position of TCC0016 (see Fig. 1). Colours represent velocity shifts expressed as values of $v_{\mathrm{lsr}}$ in $\mathrm{km} \mathrm{s}^{-1}$, where the $v_{\mathrm{lsr}}$ of OMC1 has been taken to be $12 \mathrm{~km} \mathrm{~s}^{-1}$ (see Sect. 2). Axes are labelled in arcseconds.

The results in Fig. 3 illustrate the difference between the NW region, so-called Peak 1 (Beckwith et al. 1978) and the $\mathrm{SE}$ region, Peak 2. Analysis shows that emission in Peak 1 is found on average to be $\sim 10 \mathrm{~km} \mathrm{~s}^{-1}$ more blueshifted than emission in Peak 2. This is in agreement with results in Chrysostomou et al. (1997). Comparison between our data and those of specific regions in Chrysostomou et al. may also be made, bearing in mind the different spatial resolution in the latter work (see Sect. 2). For example, the blueshifted clumps, cited in Chrysostomou et al. (1997), SW of IRc2, may be seen very clearly in our data, in a region bounded by $-15^{\prime \prime}$ to $-25^{\prime \prime} \mathrm{E}$, $-5^{\prime \prime}$ to $15^{\prime \prime} \mathrm{N}$ relative to TCC0016. Chrysostomou et al. (1997) also draw attention to red-shifted clumps in Peak 2 . These are among the most prominent features of our data in Fig. 3.

We have selected 19 distinct emitting regions, which together show numerous spatially resolved velocity structures. A finding chart, which displays velocity integrated intensity in the $\mathrm{H}_{2} v=1-0 \mathrm{~S}(1)$ line, is given in Fig. 4. The arrows in Fig. 4 show both peak velocities and position angles, discussed later in this section. Location, peak velocity, spatial displacement of peak brightness and peak velocity, position angle and values of peak brightness of the 19 regions are summarized in
Table 1. Representative examples of specific regions are given in Figs. 5 to 8 in which are shown velocity on the vertical axis, with contours to guide the eye, and the position of the zone in the $x y$-plane in arcseconds, relative to TCC0016. The colour scale superimposed on the velocity profiles shows the brightness of emission. For purposes of visualization, the brightness scale is different from figure to figure.

The principal features illustrated by data in Table 1 and in Figs. 5 to 8 may be summarized as follows. (i) There exists strong spatial correlation between flows and emission brightness. However, bright emission may also be found in the absence of detectable velocity shift (object 11, Table 1 and Fig. 8). (ii) Velocity maxima are not precisely spatially coincident with brightness maxima. Spatial displacements between velocity and brightness maxima are shown in Table 1, where values are seen to range from close to zero, e.g. 0.1 to 0.2 in regions 3 , 4, 9a, 9c, 15, 16, 18, 19 to more than $1^{\prime \prime}$. 0 in regions $2,8,10$ and 13. (iii) Brightly emitting regions may lie in close proximity. In Table 1, it may be seen that the objects $1 \mathrm{a}, 1 \mathrm{~b}$ appear very close, as do $9 \mathrm{a}, \mathrm{b}, \mathrm{c}$ and $12 \mathrm{a}, 12 \mathrm{~b}$. This is illustrated in Fig. 5, where the two brightest regions, 1a and 1b (see Fig. 4 and Table 1), lie $2500 \mathrm{AU}$ apart, for a distance of $450 \mathrm{pc}$ to 


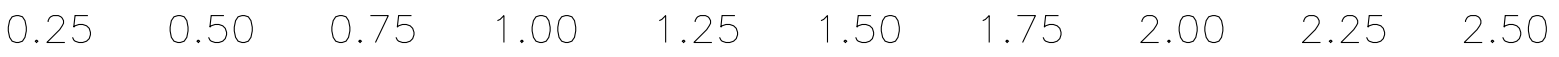

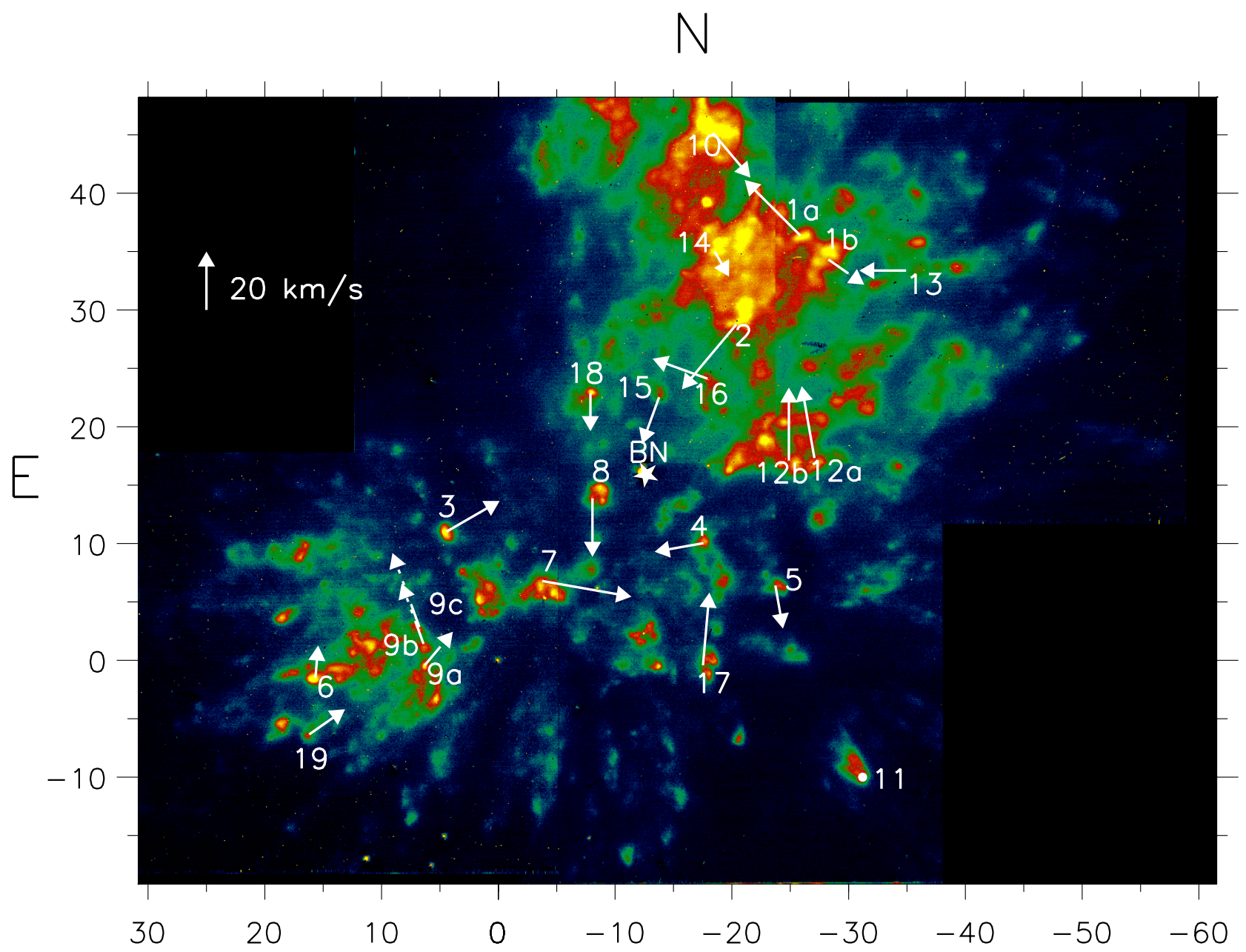

Fig. 4. Velocity integrated emission in the $\mathrm{S}(1) v=1-0 \mathrm{H}_{2}$ line in $\mathrm{OMC} 1$. Regions investigated in detail are numbered as referred to in the text and in Table 1. Arrows show the position angle of shocks and the length of arrows represents the maximum radial velocity of each feature relative to the ambient gas. Region 11 shows no measurable radial velocity, as seen in Fig. 8. More detailed data for each of the 19 regions may be found in Table 1.

Orion (O'Dell 2001). In addition, bright features tend to be superimposed on a relatively weak background emission which pervades large areas of OMC1.

The velocity extrema associated with the 19 objects shown in Table 1 are consistent with the linewidths recorded in Chrysostomou et al. (1997). The latter lie between 18 and $45 \mathrm{~km} \mathrm{~s}^{-1}$, where the present values of peak velocity lie between $\sim 10$ and $\sim 40 \mathrm{~km} \mathrm{~s}^{-1}$. We cannot of course rule out that some features in our data may represent chance superposition in the line-of-sight, rather than spatially coherent structures.

In connection with all these data, the visibility of $\mathrm{H}_{2}$ emission is strongly mediated by the local dust optical depth. In dense regions associated with star formation, the near-IR may be heavily obscured and features which are apparently weak may in reality be bright, but buried within a few hundred AU of dense material with number density $\left(n_{\mathrm{H}}+2 n_{\mathrm{H}_{2}}\right)$ which may be as high or higher than $10^{6}$ to $10^{7} \mathrm{~cm}^{-3}$ (Vannier et al. 2001;
Rosenthal et al. 2000). Thus we are likely preferentially to observe material emerging from the obscuring screen of dense gas, that is, blue-shifted from the ambient gas, rather than redshifted. Data in Table 1 illustrate this, with the large majority of flows showing locally blue-shifted emission. Region 6 , in Fig. 7, and objects 9a, b and c are exceptions to this, where objects 9 may be clearly seen as the red-shifted group in the velocity field illustrated in Fig. 3.

Data are analyzed below to show that fast components of flows, such as those in Figs. 5 to 8, are local to the regions observed and do not represent components of a large-scale flow. This is in contrast to data for high velocity Herbig-Haro objects whose motion is represented by a general outflow with an origin close to BN-IRc2 (Lee \& Burton 2000; Doi et al. 2002; Rosado et al. 2001). Implicit in our subsequent discussion is the assumption that shocks are responsible for the major part of the emission observed. $\theta^{1}$ Ori $\mathrm{C}$ in the Trapezium cluster generates 
Table 1. Location of peak brightness, peak velocity, spatial displacement of peak velocity and peak brightness, position angle and value of peak brightness for the 19 objects selected here. The location of each object is measured in arcseconds relative to TCC0016, located at $05^{\mathrm{h}} 35^{\mathrm{m}} 14^{\mathrm{s}} .91$, $-05^{\circ} 22^{\prime} 39$ !' 31 (J2000). The peak velocity is the peak radial velocity with respect to the velocity of the local ambient gas, the latter estimated from the average velocity of weak emission in the vicinity of fast flows. The position angle is measured clockwise from the north, and the peak brightness is given in counts $\mathrm{s}^{-1}$ where 1 count s $\mathrm{s}^{-1}$ corresponds to $8.7 \pm 0.4 \times 10^{-6} \mathrm{~W} \mathrm{~m}^{-2} \mathrm{sr}^{-1}$.

\begin{tabular}{|c|c|c|c|c|c|c|c|c|}
\hline \multirow{2}{*}{$\begin{array}{l}\text { Object } \\
1 \mathrm{a}\end{array}$} & \multicolumn{4}{|c|}{$\begin{array}{c}\text { Location } \\
\text { relative to TCC0016 }\end{array}$} & \multirow{2}{*}{$\begin{array}{c}\begin{array}{c}\text { Peak velocity } \\
/ \mathrm{km} \mathrm{s}^{-1}\end{array} \\
-27\end{array}$} & \multirow{2}{*}{$\begin{array}{c}\begin{array}{c}\text { Displacement } \\
\text { /arcsec }\end{array} \\
0.4\end{array}$} & \multirow{2}{*}{$\begin{array}{c}\text { Position angle } \\
315^{\circ}\end{array}$} & \multirow{2}{*}{$\begin{array}{c}\begin{array}{c}\text { Peak brightness } \\
\text { /counts s }\end{array} \\
2.53\end{array}$} \\
\hline & $25 . .7$ & $\mathrm{~W}$ & $36 ! " 2$ & $\mathrm{~N}$ & & & & \\
\hline $1 \mathrm{~b}$ & $28 . \prime 1$ & $\mathrm{~W}$ & $34{ }^{\prime \prime} 1$ & $\mathrm{~N}$ & 15 & 0.7 & $125^{\circ}$ & 2.75 \\
\hline 2 & $20 ! 3$ & $\mathrm{~W}$ & $28 . .6$ & $\mathrm{~N}$ & -29 & 1.2 & $220^{\circ}$ & 2.47 \\
\hline 3 & 4.' 4 & E & $11^{\prime \prime} 0$ & $\mathrm{~N}$ & -21 & 0.2 & $60^{\circ}$ & 2.91 \\
\hline 4 & $17 . .2$ & W & $10 !^{\prime} 0$ & $\mathrm{~N}$ & -17 & 0.2 & $260^{\circ}$ & 2.07 \\
\hline 5 & $23 . " 3$ & W & $6 . " 4$ & $\mathrm{~N}$ & -15 & 0.4 & $170^{\circ}$ & 1.75 \\
\hline 6 & $15^{\prime \prime} 6$ & $\mathrm{E}$ & 1..5 & S & 11 & 0.5 & $5^{\circ}$ & 3.43 \\
\hline 7 & $3 . .7$ & $\mathrm{~W}$ & 6." 8 & $\mathrm{~N}$ & -31 & 0.4 & $100^{\circ}$ & 2.64 \\
\hline 8 & $8 . .0$ & $\mathrm{~W}$ & $133^{\prime \prime} 8$ & $\mathrm{~N}$ & -20 & 1.1 & $180^{\circ}$ & 2.37 \\
\hline $9 \mathrm{a}$ & $6 . ' 3$ & $\mathrm{E}$ & $0 . ' 4$ & S & 15 & 0.1 & $40^{\circ}$ & 2.36 \\
\hline $9 b$ & 6." 4 & $\mathrm{E}$ & 1." 4 & $\mathrm{~N}$ & 22 & 0.3 & $340^{\circ}$ & 1.73 \\
\hline $9 \mathrm{c}$ & 6.7 & $\mathrm{E}$ & 2.8 & $\mathrm{~N}$ & 27 & 0.1 & $340^{\circ}$ & 1.62 \\
\hline 10 & $18^{\prime \prime} 3$ & $\mathrm{E}$ & $44 . \prime 9$ & $\mathrm{~N}$ & -20 & 1.3 & $140^{\circ}$ & 3.00 \\
\hline 11 & 30.9 & W & 10.0 & S & 0 & - & - & 1.75 \\
\hline $12 \mathrm{a}$ & $26 . .9$ & $\mathrm{~W}$ & $17 ! .2$ & $\mathrm{~N}$ & -25 & 0.7 & $350^{\circ}$ & 2.04 \\
\hline $12 b$ & $24 . .7$ & W & $17 . .0$ & $\mathrm{~N}$ & -25 & 0.6 & $0^{\circ}$ & 2.32 \\
\hline 13 & $34 . .7$ & W & $33 \prime .2$ & $\mathrm{~N}$ & -16 & 1.0 & $270^{\circ}$ & 1.79 \\
\hline 14 & $18 . " 4$ & W & $344^{\prime \prime} 8$ & $\mathrm{~N}$ & -10 & 0.8 & $150^{\circ}$ & 2.28 \\
\hline 15 & $133^{\prime \prime} 7$ & W & $22^{\prime \prime} 4$ & $\mathrm{~N}$ & -17 & 0.2 & $200^{\circ}$ & 0.99 \\
\hline 16 & $17{ }^{\prime \prime} 8$ & W & 20.5 & $\mathrm{~N}$ & -20 & 0.2 & $290^{\circ}$ & 1.23 \\
\hline 17 & $17 . .1$ & W & $0 . .4$ & S & -25 & 0.9 & $5^{\circ}$ & 2.01 \\
\hline 18 & 7!. 8 & W & $22^{\prime \prime} 8$ & $\mathrm{~N}$ & -13 & 0.1 & $180^{\circ}$ & 1.94 \\
\hline 19 & $16 .^{\prime \prime} 1$ & $\mathrm{E}$ & 6.3 & $\mathrm{~S}$ & -15 & 0.1 & $55^{\circ}$ & 1.67 \\
\hline
\end{tabular}

a very intense far-UV radiation field which will also contribute to excitation of $\mathrm{H}_{2}$ into vibrationally excited states. Detailed models (Störzer \& Hollenbach 1999) have been devised describing the action of radiation fields corresponding to that of $\theta^{1}$ Ori $\mathrm{C}$, falling upon dense gas. These models show that the maximum brightness in the $v=1-0 \mathrm{~S}(1)$ line formed by a fluorescence mechanism including "photoevaporation" (Henney \& O'Dell 1999) does not exceed $10-15 \%$ of the total brightness observed, for example in region 6 (Fig. 7), one of the closest lying regions to the Trapezium cluster. Thus photon excitation is a minor though non-negligible contributor to the total brightness. This issue is discussed in detail in Kristensen et al. (2003).

Data in Figs. 5-8 present views of flows, embedded in the local gas and travelling at different angles to the line-of-sight (l.o.s.). Referring to Table 1, those flows for which the displacement between maximum velocity and brightness is small, may be interpreted as flows close to the l.o.s., such as object 4, Fig. 6. One zone, region 11, shows no observable radial velocity (Fig. 8), which we interpret therefore as a flow in the plane of the sky. If the region is of uniform density, and of homogeneous magnetic field, a flow travelling in the l.o.s. would show maximum brightness spatially coincident with maximum velocity. We interpret the displacement of maxima in velocity and brightness in terms of viewing angle effects in flows travelling at an angle to the 1.o.s.. The tip of a bow-shaped shock seen at an angle to the 1.o.s. yields weak emission, because of low column density of excited $\mathrm{H}_{2}$. Progressing further along the shock in projection, a combination of column density and optimum shock excitation conditions would be expected to yield a maximum in brightness in the plane of the sky, as observed. Thus the maximum in emission and the maximum in velocity lie on a vector defining the orientation of the shock in the plane of the sky, that is, its position angle. Position angles were determined accordingly and are shown in Table 1 . Spatial uncertainties in the position of the maximum velocity, and the presence of more than one maximum in either or both velocity and brightness, result in an uncertainty in the derived position angle of typically $\pm 5^{\circ}$ and no more than $\pm 10^{\circ}$. Figure 4 shows estimated position angles of each of the 19 features (save feature 11), through arrows attached to each zone, where the direction of the arrow gives the position angle of the flow. The length of the arrow gives the maximum radial component of the shock velocity, where this is estimated by assuming that the base of the shock is associated with the local velocity of weak emission in the vicinity of the fast component of the flow.

There is no general direction for the shocks illustrated in Fig. 4; arrows appear to point in random directions. Shocks are therefore in general intrinsic to the clumps of gas studied. We propose that at least some of the clumps reported here contain protostars, where characteristic outflow (André et al. 1993; Evans 1999; Eislöffel et al. 2000) creates the strongly emitting shocked regions. The clumps are themselves bathed in a background wind from BN-IRc2 which permeates OMC1. This, 

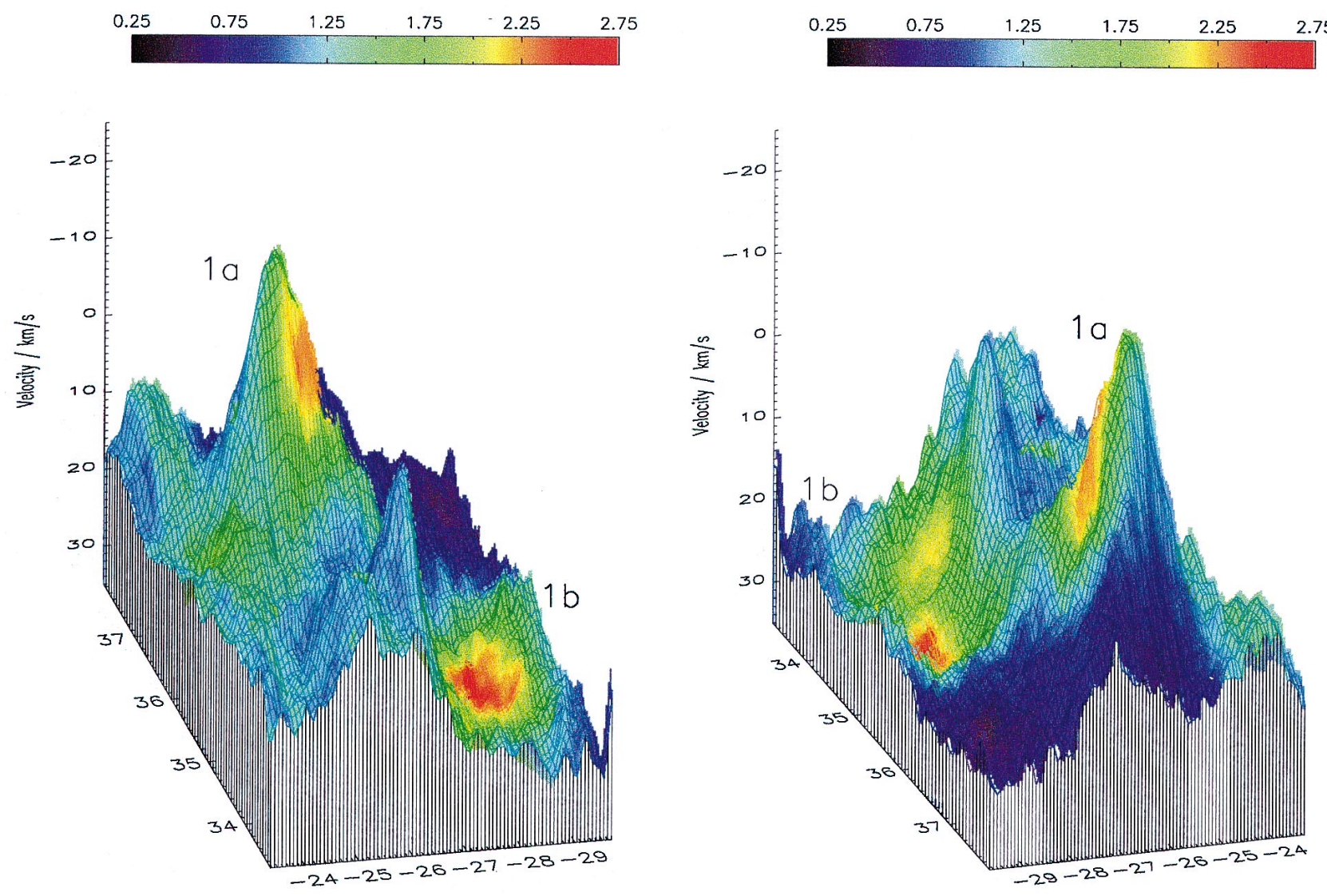

Fig. 5. Two views of the brightly emitting zones labeled 1a and $1 \mathrm{~b}$ in Fig. 4. The vertical axis shows the velocity relative to the local standard of rest $\left(v_{\mathrm{lsr}}\right)$. The horizontal plane is the plane of the sky and the centre of this image lies $266^{\prime \prime} 9 \mathrm{~W}$ and $35^{\prime \prime} .6 \mathrm{~N}$ relative to TCC0016. Colours represent brightness, with the scale shown by the colour bar, in counts per pixel per second (see text and caption to Table 1). Contours are of velocity, included to guide the eye. The viewing angle in the lhs image is $40^{\circ}$ in the vertical plane and $20^{\circ}$ in the horizontal, measured anti-clockwise, that is, as seen from the South-East. The rhs image shows a viewing angle of $30^{\circ}$ in the vertical plane and $150^{\circ}$ clockwise in the horizontal plane, that is, as seen from the North-West.

with the radiation field of the Trapezium stars, gives rise to pervasive but relatively weak $\mathrm{H}_{2}$ excitation.

To develop this model for the strongly emitting zones, reference may be made to objects $1 \mathrm{a}$ and $\mathrm{b}$ (Figs. 4 and 5). As noted, $1 \mathrm{a}$ and $\mathrm{b}$ lie $\sim 2500 \mathrm{AU}$ apart in projection. If the bright zones are the Mach disks of a bipolar outflow, a protostar would be found roughly midway in velocity between the regions of bright emission, with each outflow having a radial velocity component of $\sim 25 \mathrm{~km} \mathrm{~s}^{-1}$. Other regions also show closelylying bright zones. For example region 8 (details not shown here) is nearly as striking as region 1 in this respect, with the two zones lying only $\sim 450 \mathrm{AU}$ apart in projection. Other objects listed in Table 1 show however only one region of very bright emission, as in objects 4 and 6, Figs. 6 and 7. This may arise if one side of the bipolar flow is obscured by dust.

If some zones recorded in Table 1 represent sites of star formation, how do they compare with more conventional protostellar regions and is it possible to place such objects within the standard classification of protostellar zones (André et al. 1993; Evans 1999)? We find below that classification in standard terms is not possible, in part because of the lack of appropriate observational data and in part because the Orion objects simply do not closely resemble objects previously reported.

The properties of protostellar zones, to some degree as characterized through $\mathrm{H}_{2}$ emission, may be summarized as follows:

(i) The density in the circumstellar envelope may be typically $10^{4}$ to $10^{5} \mathrm{~cm}^{-3}$ at distances of (say) a few hundred to a few thousand AU or more from the protostars (Evans 1999).

(ii) Regions containing shocked $\mathrm{H}_{2}$ tend to be highly localised (Eislöffel et al. 2000) and the emission brightness is at least a factor 5 and typically an order of magnitude or more below that seen here (Davis et al. 2001; Davis et al. 2002; Buckle et al. 1999); ignoring any differences in NIR opacity.

(iii) With regard to values of velocity, in a set of 9 protostellar zones, Davis et al. (2001) reports projected $\mathrm{H}_{2}$ flow velocities ranging from 5 to $40 \mathrm{~km} \mathrm{~s}^{-1}$ for low velocity components.

(iv) $\mathrm{H}_{2}$ emission has been observed to start as close as a few hundred AU from the protostar and outflows have projected lengths which vary between $500 \mathrm{AU}$ to more than $10^{4} \mathrm{AU}$ (Davis et al. 2002; Davis et al. 2001; Richer et al. 2000). 


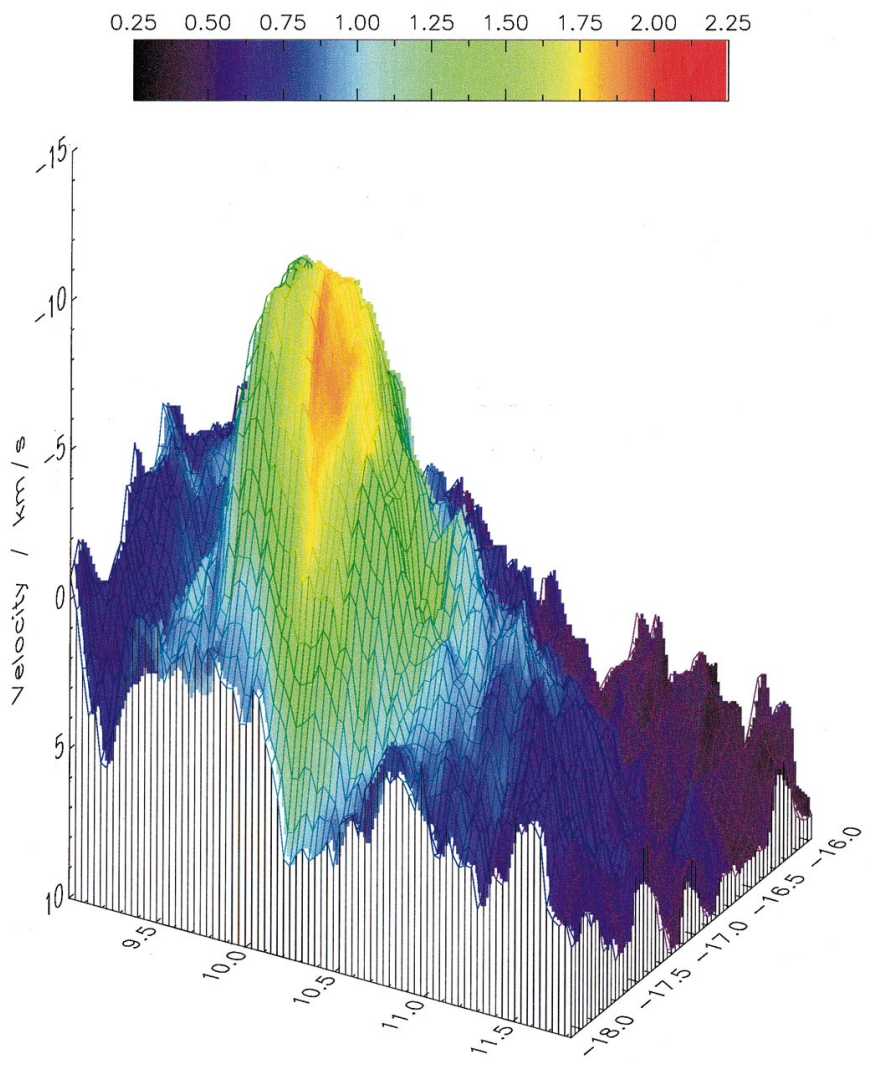

Fig. 6. The brightly emitting zone labeled 4 in Fig. 4. The centre of this image lies $17^{\prime \prime} 6 \mathrm{~W}$ and $10^{\prime} .1 \mathrm{~N}$ relative to TCC0016. Otherwise as for Fig. 5, but with a viewing angle of $25^{\circ}$ in the vertical plane and $120^{\circ}$ clockwise in the horizontal plane, that is, as seen from the North-West.

(v) The observational properties of the very earliest "Class 0" objects (André et al. 1993; Evans 1999) have been summarised in a number of articles e.g. Bachiller (1996); Ciardi et al. (2003). In brief, Class 0 objects are invisible at NIR wavelengths in the direction of the objects themselves, have a sub$\mathrm{mm}(\lambda>350 \mu \mathrm{m})$ to bolometric luminosity ratio of $\geq 0.5 \%$, possess a spectral energy distribution characterised by a modified blackbody with a bolometric temperature $<70 \mathrm{~K}$ and show molecular outflows, which may often be visible in $\mathrm{H}_{2}$ NIR emission far from the highly obscured core. Class 0 protostars also possess a circumstellar envelope more massive than the protostar itself. For the present for the Orion objects we unfortunately lack standard ingredients for a description of the nature of protostellar zones, namely spectral energy distributions (SEDs) and bolometric luminosities.

We review the Orion objects briefly below, with reference as appropriate to the properties (i)-(v) outlined above.

(i) shock models (Vannier et al. 2001; Le Bourlot et al. 2002) show that in order to reproduce the high $\mathrm{H}_{2}$ emission brightness recorded in Table 1, magnetic C-type shocks are required of velocity 25 to $30 \mathrm{~km} \mathrm{~s}^{-1}$ impinging on preshock gas of density $10^{6} \mathrm{~cm}^{-3}$ or more. Thus the densities of gas in the 19 regions in Table 1 are more than an order of magnitude higher than in conventional sources. A density of $10^{6} \mathrm{~cm}^{-3}$ is in fact typical of massive cores, associated with the formation

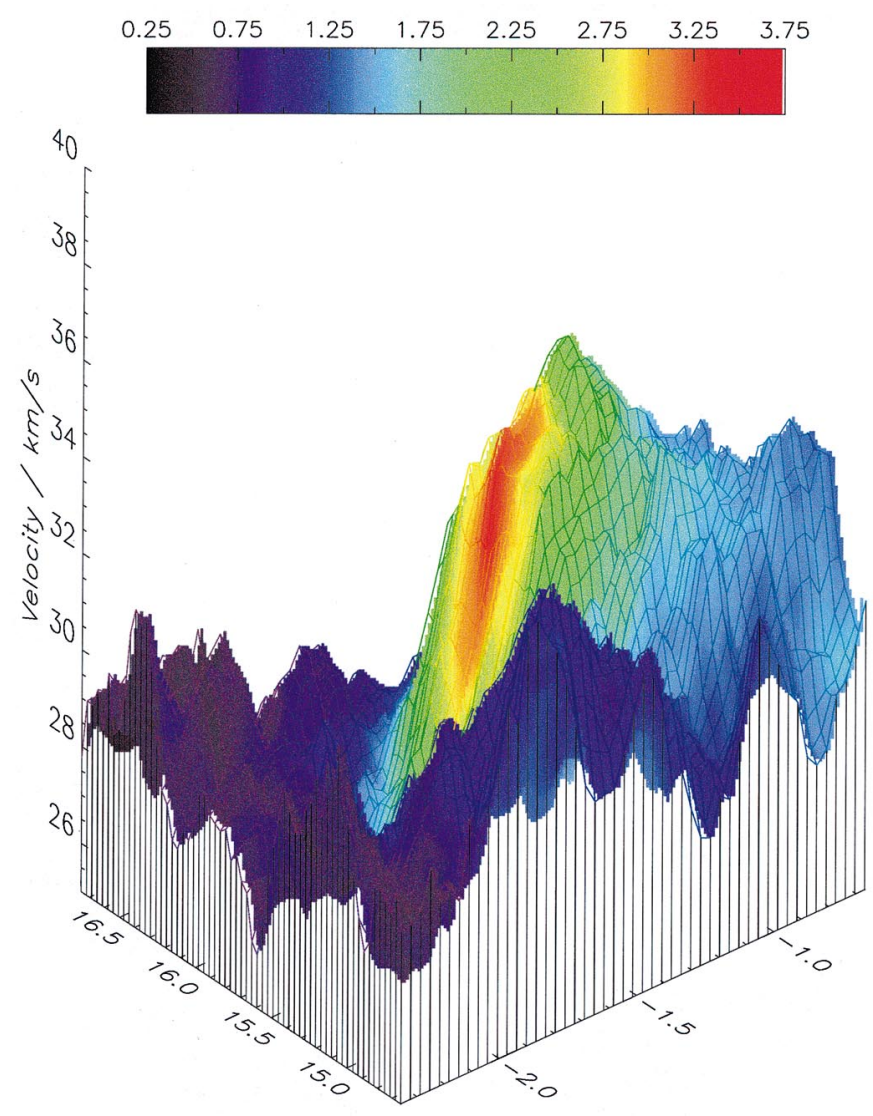

Fig. 7. The brightly emitting zone labeled 6 in Fig. 4. The centre of this image lies $155^{\prime \prime} 7 \mathrm{E}$ and 1". $8 \mathrm{~S}$ relative to TCC0016. Otherwise as for Fig. 5, but with a viewing angle of $30^{\circ}$ in the vertical plane and $50^{\circ}$ clockwise in the horizontal plane, that is, as seen from the South-West. Note that this flow is redshifted relative to the ambient gas.

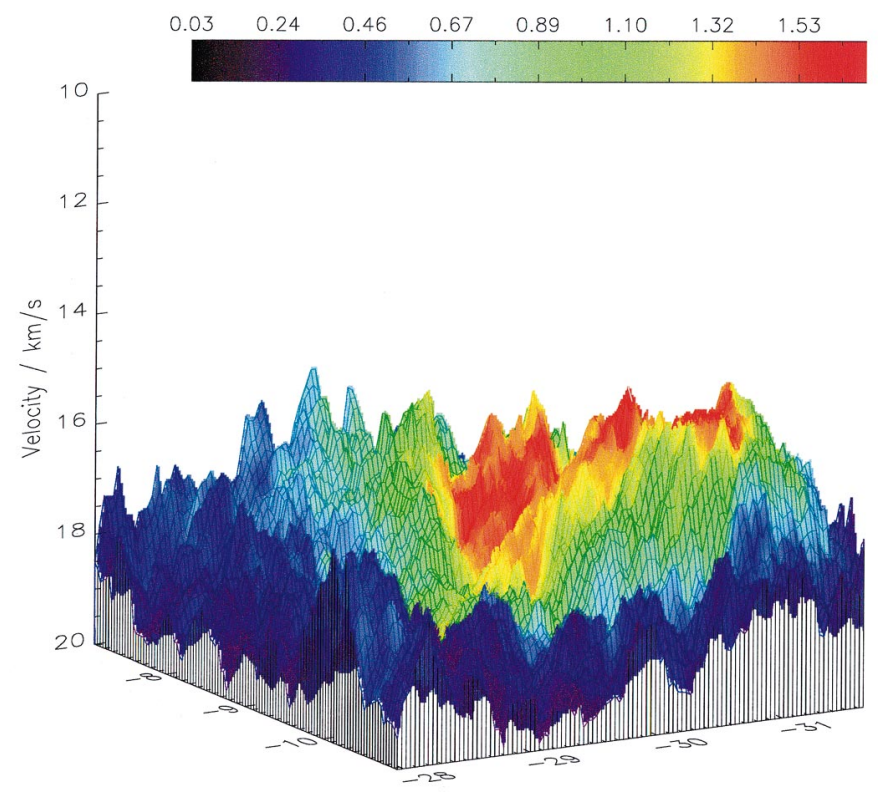

Fig. 8. The brightly emitting zone labeled 11 in Fig. 4. The centre of this image lies 30.' $4 \mathrm{~W}$ and 9.'1S relative to TCC0016. Otherwise as for Fig. 5, but with a viewing angle of $20^{\circ}$ in the vertical plane and $30^{\circ}$ anti-clockwise in the horizontal plane, that is, as seen from the South-East. 
of clusters of massive stars (Evans 1999). The amount of material in motion in the most massive clump observed here, that is, in region 6 (Vannier et al. 2001), is however of the order of only $\sim 0.1 M_{\odot}$ (Vannier et al. 2001). Since this is two orders of magnitude or more lower than the mass associated with outflows from massive star-forming regions (Churchwell 1997), massive star formation in these Orion clumps does not seem plausible, at any rate on the basis of our present understanding. Moreover, the column densities associated with the emitting regions of $10^{23} \mathrm{~cm}^{-2}$ to a few times $10^{23} \mathrm{~cm}^{-2}$ are typical of low mass protostellar zones (Davis et al. 2001).

(ii) Bright emission is seen here from zones extending over a greater range of angle than in conventional regions, that is, the outflows are not well collimated, but maintain a significant isotropic component. There is also evidence of complex flows with more than one component, for example in region 1 which possesses a subsidiary flow close to where a protostar may lie. In addition, the view of region 1 in the rhs figure presents evidence for two outflows. Complexity of this nature is also clearly apparent in region 8. Multipolar, e.g. quadrupolar, outflows are known in other regions (Palacios \& Eiroa 1999) where such action tends to be attributed to the presence of more than one protostar (Gueth et al. 2001; Lucas et al. 2000) - or is typical of massive star formation (Churchwell 1997).

(iii) Shock velocities, recorded (in projection) as peak velocities in Table 1, cover the range encountered in protostellar zones. The energy flux in the shocks is however more than an order of magnitude greater, given the higher densities involved. Detailed modelling using C-type shock codes, in conjunction with velocities from the present data, could lead in future work to estimates of the mass loss rate from the regions observed. These estimates in turn may yield estimates of accretion rates (Richer et al. 2000) and allow comparison to be made with conventional Class 0 or Class 1 sources in conjunction with SEDs and bolometric luminosities (Jayawardhana et al. 2001; Calvet et al. 2000).

(iv) $\mathrm{H}_{2}$ emission is observed in projection all around the possible position of a protostar. This might suggest that the extinction is weak even very close to the protostar, in marked contrast to isolated protostellar zones. In Orion however, as mentioned above, some of the weaker emission arises through shocks associated with the pervasive outflow from BN/IRc2 (O'Dell 2001) and also from photon-induced processes from $\theta^{1}$ Ori C, the dominant source in the Trapezium cluster (Störzer \& Hollenbach 1999). Thus the morphology of the $\mathrm{H}_{2}$ emission may be regarded as a superposition of local outflows, yielding $\mathrm{H}_{2}$ emission intrinsic to protostars, and a general weaker background, which is not directly associated with protostars buried within OMC1. In connection with the presence of protostars within $\mathrm{OMC1}$, it is interesting to note that data obtained very recently (late November 2002) using the NAOS-CONICA adaptive optics system on the European Southern Observatory Very Large Telescope show that region 6 contains a compact object of diameter of no more than $140 \mathrm{AU}$, emitting in the continuum. This is a good diagnostic of a partly unveiled solar system size disk.

(v) In connection with comparison with Class 0 objects, the high extinction associated with Class 0 may be recon- ciled with our observations if, as suggested above, only the brightest emission is intrinsic to buried protostars. In addition, as noted earlier, the mass of circumstellar material in OMC1 clumps does not exceed $\sim 0.1 M_{\odot}$ (Vannier et al. 2001), and a class 0 characteristic would imply protostars of this mass or less. However, material will have been removed both by the wind from BN-IRc2 (O'Dell 2001) and, by analogy with the later "proplyds", by photoevaporation due to the Trapezium stars (O'Dell 2001; Störzer \& Hollenbach 1999; Henney \& O'Dell 1999). This suggests that the mass of the circumstellar material may originally have been greater than estimates of the current mass in Vannier et al. (2001). At all events, these removal processes will limit the accretion that can take place.

The above comparison between the general properties of objects in Table 1 and the known general characteristics of early stages of star formation, the latter for isolated protostars in the absence of perturbations from nearby massive stars, reveals both similarities and differences. The high brightness (see Table 1) of the Orion objects requires abnormally high densities and high energy flux in the associated flows, whereas flow velocities and the physical scale of shocked regions seem typical of early stages of star formation. The properties of the present regions suggest that the Orion objects may represent a more energetic and less structured stage of star formation than has previously been recorded. If this is so, objects may settle down to less unruly behaviour or they may represent a different stellar lineage, eaten away on one side by the powerful radiation field of the Trapezium stars to the south and blasted continuously on the other face by the fast wind from BN-IRc2.

Acknowledgements. DF and MG would like to acknowledge the support of the Aarhus Centre for Atomic Physics (ACAP), funded by the Danish Basic Research Foundation. DF would also like to thank the Observatoire Paris-Meudon for support during the period in which this work was performed. JLL would like to acknowledge the support of the PCMI National Program funded by the CNRS. We would also like to thank the Directors and Staff of the CFHT for making possible the observations reported in this paper.

\section{References}

Allen, D. A., \& Burton, M. G. 1993 Nature, 363, 54

André, P., Ward-Thompson, D., \& Barsony, M. 1993, ApJ, 406, 122

Bachiller, R. 1996, ARA\&A, 34, 111

Beckwith, S., Persson, S. E., Neugebauer, G., \& Becklin, E. E. 1978, ApJ, 223, 464

Bragg S. L., Smith W. H., \& Brault J. W. 1982, ApJ, 263, 999

Buckle, J. V., Hatchell, J., \& Fuller. G. A. 1999, A\&A, 348, 584

Calvet, N., Hartmann, L., \& Strom. S. E. 2000, Protostars and Planets IV, ed. V. Mannings, A. P. Boss, \& S. S. Russeell (Tucson: University of Arizona Press)

Chen, H., Bally, J., O’Dell, C.R., et al. 1998, ApJ, 492, L173

Ciardi, D. R., Telesco, C. M., Williams, J. P., et al. 2003, ApJ, 585, 392

Chrysostomou, A., Burton, M. G., Axon, D. J., et al. 1997, MNRAS, 289,605

Churchwell, E. 1997, Massive Star Formation: Observational Constraints, in: Herbig Haro Flows and the Birth of Low Mass Stars, ed. B. Reipurth, \& C. Bertout, IAU Symp. 182 (Kluwer Acadaemic Publ.) 
Clénet, Y., Le Coarer, E., Joncas, G., et al. 2002, PASP, 114, 563

Davis, C. J., Ray, T. P., Desroches, L., \& Aspin, C. 2001, MNRAS, 326,524

Davis, C. J., Stern, L., Ray, T. P., \& Chrysostomou, A. 2002, A\&A, 382,1021

Doi, T., O’Dell, C. R., \& Hartigan, P. 2002, AJ, 124, 445

Eislöffel, J., Mundt, R., Ray T. P., \& Rodriguez, L. F. 2000, Protostars and Planets IV, ed. V. Mannings, A. P. Boss, \& S. S. Russeell (Tucson: University of Arizona Press)

Evans, N. J. 1999, ARA\&A, 37, 311

Ferland, G. J. 2001, PASP, 113, 41

Gueth F., Schilke P., \& McCaughrean M. J. 2001, A\&A, 375, 1018

Henney, W. J., \& O’Dell, C. R. 1999, AJ, 118, 2350

Jayawardhana, R., Hartmann, L., \& Calvet, N. 2001, ApJ, 548, 310

Kristensen, L. E., Gustafsson, M., Field, D., et al. 2003, A\&A, to appear

Lee J.-K., \& Burton M. G. 2000, MNRAS, 315, 11

Le Bourlot, J., Pineau des Forêts, G., Flower, D. R., \& Cabrit, S. 2002, MNRAS, 332, 985
Lucas P. W., Blundell K. M., \& Roche P. F. 2000, MNRAS, 318, 526

Luhman, K. L., Rieke, G. H., Young, E. T., et al. 2000, ApJ, 540, 1016 O’Dell, C. R. 2001, ARA\&A, 39, 99

Palacios, J., \& Eiroa, C. 1999, A\&A, 346, 233

Richer, J. S., Shepherd, D. S., Cabrit, S., Bachiller, \& Churchwell, R. E. 2000, in Protostars and Planets IV, ed. V. Mannings, A. P. Boss, \& S. S. Russell (Tucson: University of Arizona Press)

Rosado, M., de la Fuente E., Arias, L., Raga, A., \& Le Coarer, E., 2001, AJ, 122, 1928

Rosenthal, D., Bertoldi, F., \& Drapatz, S. 2000, A\&A, 356, 705

Salas, L., Rosado, M., Cruz-Gonzáles, I., et al. 1999, ApJ, 511, 822

Schild, H., Miller, S., \& Tennyson, J. 1997, A\&A, 318, 608

Schultz, A. S. B., Colgan, S. W. J., Erickson, E. F., et al. 1999, ApJ, 511,282

Stolovy, S. R., Burton, M. G., Erickson, E. F., et al. 1998, ApJ, 492, L151

Störzer, H., \& Hollenbach, D. J. 1999, ApJ, 515, 669

Sugai, H., Kawabata, H., Usuda, T., et al. 1995, ApJ, 442, 674

Vannier, L., Lemaire, J. L., Field, D., et al. 2001, A\&A, 366, 651 